University of South Carolina

Scholar Commons

$10-2004$

\title{
Lower Extremity Muscle Strength and Risk of Self-Reported Hip or Knee Osteoarthritis
}

Jennifer M. Hootman

Shannon J. FitzGerald

Carol A. Macera

Steven N. Blair

University of South Carolina - Columbia, sblair@mailbox.sc.edu

Follow this and additional works at: https://scholarcommons.sc.edu/

sph_epidemiology_biostatistics_facpub

Part of the Public Health Commons

\section{Publication Info}

Published in Journal of Physical Activity and Health, Volume 1, Issue 4, 2004, pages 321-330. Hootman, J. M., FitzGerald, S., Macera, C. A., \& Blair, S. N. (2004). Lower extremity muscle strength and risk of self-reported hip or knee osteoarthritis Journal of Physical Activity and Health, 1(4), 321-330. (c) Journal of Physical Activity and Health, 2004, Human Kinetics inclusion in Faculty Publications by an authorized administrator of Scholar Commons. For more information, please contact digres@mailbox.sc.edu. 


\title{
Lower Extremity Muscle Strength and Risk of Self-Reported Hip or Knee Osteoarthritis
}

\author{
Jennifer M. Hootman, Shannon FitzGerald, \\ Carol A. Macera, and Steven N. Blair
}

\begin{abstract}
Purpose: The purpose of this study was to investigate the gender-specific longitudinal association between quadriceps strength and self-reported, physician-diagnosed hip or knee osteoarthritis (OA). Methods: Subjects were 3081 community-dwelling adults who were free of OA, joint symptoms and injuries, completed a maximum treadmill exercise test, had isokinetic knee extension and flexion and isotonic leg press strength measurements taken at baseline and returned at least one written follow-up survey. Multivariate logistic regression was used to estimate odds ratios and $95 \%$ confidence intervals. Results: Women with moderate or high isokinetic quadriceps strength had a significantly reduced risk (55\% to $64 \%$ ) of hip or knee OA. A similar, nonsignificant trend was noted among men. Moderate isotonic leg press strength was protective for hip or knee osteoarthritis among men only. Conclusions: These results suggest that quadriceps weakness is an independent and modifiable risk factor for lower extremity OA, particularly among women.
\end{abstract}

Key Words: isokinetic, muscle weakness, degenerative arthritis, exercise, prevention

Quadriceps muscle weakness is a well-established clinical feature of knee osteoarthritis $(\mathrm{OA})^{1-4,5}$ and the pelvic and thigh muscle strength of hip osteoarthritis patients have been found to be $18 \%$ to $31 \%$ weaker compared to healthy controls. ${ }^{6}$ Patients report "giving way" and global lower extremity weakness during activities of daily living and clinicians note quadriceps muscle atrophy and muscle weakness on physical examination. Muscle weakness is also associated with increased functional limitation and disability and decreased balance among persons with osteoarthritis. ${ }^{2,47,8}$ Studies show that muscle strengthening programs, either through physical therapy interventions ${ }^{9-11}$ or general resistance training physical activity programs, ${ }^{12-15}$ are beneficial for people with osteoarthritis and improve arthritisrelated symptoms such as pain, stiffness, and physical function.

Hootman is with the National Center for Chronic Disease Prevention and Health Promotion, Centers for Disease Control and Prevention, Atlanta, GA 30341. FitzGerald and Blair are with the Centers for Integrated Health Research, Cooper Institute, Dallas, TX 75230. Macera is with the Department of Epidemiology and Biostatistics, San Diego State University, San Diego, CA 92182. 
Less is known about the association between quadriceps muscle weakness and incident lower extremity osteoarthritis. Slemenda et al. ${ }^{16}$ postulated that quadriceps weakness might be an independent risk factor for knee osteoarthritis and, in a crosssectional study, reported that quadriceps weakness, but not hamstring weakness, was common in persons with radiographic knee osteoarthritis. In a later longitudinal study, women with no knee pain or muscle atrophy who developed radiographic knee osteoarthritis had $15 \%$ to $18 \%$ lower quadriceps strength at baseline than those without knee osteoarthritis. ${ }^{17}$ This relationship was not demonstrated among men, however. To date, no longitudinal studies have been published investigating the relationship between muscle weakness and hip osteoarthritis.

Previous studies investigating the association between quadriceps weakness and lower extremity osteoarthritis have several limitations. First, only one longitudinal study has been reported ${ }^{17}$ and this study had a relatively short follow-up period (approximately 30 months). Second, most subjects in previous studies were elderly at baseline. Older subjects could already have age-related muscle changes such as sarcopenia ${ }^{18}$ as well as have other comorbid conditions that affect muscle strength. Third, most studies had small-to-moderate sample sizes which might limit the power to detect associations especially among subgroups such as women. Fourth, no studies adequately controlled for the subject's physical activity or fitness level, an important covariate to consider in studies of muscle strength. Therefore, the purpose of this study was to investigate the longitudinal association between quadriceps muscle weakness and incident self-reported, physician-diagnosed hip or knee osteoarthritis and to identify differences in this relationship by gender.

\section{Materials and Methods}

\section{Subjects}

The subjects are participants in an epidemiological study of health and fitness specifically designed to investigate the relationships among physical activity, cardiorespiratory fitness, and disease outcomes. Subjects are self- or employerreferred to the study for preventive medical examinations or health and nutrition counseling. The level of educational attainment is high ( $80 \%$ have a college degree) and most (97\%) are Caucasian. At the baseline examination, subjects complete a comprehensive health history questionnaire, undergo a complete physical examination by a physician, and have laboratory blood analyses and a maximal exercise stress test on a treadmill. Between 1980 and 1989, a self-selected subset of subjects ( $n=5614)$ also participated in musculoskeletal strength testing during their baseline examination. Subjects who complete the baseline examination are followed via periodic mailed health surveys approximately every $5 \mathrm{y}$. All subjects provided written informed consent and the research protocol is reviewed annually by an institutional review board.

\section{Inclusion Criteria}

Subjects were excluded at baseline if they reported any joint pain, stiffness or swelling, arthritis, gout, joint replacement, previous hip or knee injury on a health history questionnaire or did not reach $85 \%$ of their age-predicted maximum heart 
rate on a maximal exercise treadmill test. In addition, we included only adults age 20 y or older who returned the 1986 survey and at least one other follow-up survey in 1990, 1995, or 1999. After exclusions, a total of 2422 men and 659 women met all study criteria.

\section{Outcome Definition}

Hip or knee osteoarthritis was determined according to a positive response, on at least one of the three follow-up surveys, to the following question "Has a doctor ever told you that you have hip or knee osteoarthritis?" This question has shown moderate agreement $(\kappa=0.68)$ with medical record documentation of osteoarthritis. ${ }^{19}$

\section{Laboratory Strength Measurements}

The strength evaluation consisted of an isolated bilateral knee flexion and extension strength test at $60^{\circ}$ per second on an isokinetic dynamometer (Cybex 340, Chattecx Corp., Chattanooga, TN) and a global measure of bilateral lower extremity strength using a 1 repetition maximum (1 RM) isotonic leg press test on a Universal leg press machine (Universal Gym Equipment, Cedar Rapids, IA). All tests were conducted by a trained research assistant using standardized testing protocols. On entering the laboratory, subjects were questioned about current injuries and health conditions that would preclude strength testing and completed a 2.5 min warm-up on a stationary bicycle. For the isokinetic testing, subjects were seated on the Cybex 340 and the instrument was aligned, the subject stabilized, and gravity correction done according to the manufacturer's instructions. The dominant leg was tested first followed by the nondominant leg. Subjects completed 4 warm-up trial repetitions at $60^{\circ}$ per second with the first 3 repetitions submaximal (70 to $80 \%$ ) effort and the last one maximal effort. Following the 4 warm-up repetitions, 3 test repetitions at maximal effort were recorded and the procedure was repeated on the opposite extremity. For the isotonic $1 \mathrm{RM}$ leg press, subjects were seated with the ball of the foot on the crease of the footplate of the leg press machine with the knees at $60^{\circ}$ to $65^{\circ}$ of flexion measured by goniometer. Subjects were instructed to slowly extend their legs without hyperextension and slowly return to the starting position. The initial weight was $100 \%$ of the subject's body weight. Additional weight was added in 5 to 10 pound increments depending on the subject's age, gender, physical activity history, and fitness level. The procedure was repeated until the subject reached maximal load. The protocol was designed to achieve maximum load in 5 trials.

\section{Covariate Definitions}

The subject's age and gender were recorded at baseline on the medical history questionnaire. During the physical exam, body weight and height were measured and recorded and body mass index was calculated using the formula weight in kilograms/height in meters ${ }^{2}$. Information on musculoskeletal injury history was obtained on the baseline questionnaire as well as on the 1986 survey. Subjects who reported any hip or knee injury at either timepoint were classified as having a positive injury history and were excluded as described above. Physical activity level was determined from self-reported regular exercise patterns and was clas- 
sified as sedentary (no regular exercise), low (walk/jog $<10 \mathrm{mi} / \mathrm{wk}$ ), moderate (walk/jog 10 to $20 \mathrm{mi} / \mathrm{wk}$, high (walk/jog 20+ mi per wk) and other (participation in some regular activity but not walking or jogging). Cardiorespiratory fitness was determined from a maximal exercise test using a modified Balke protocol ${ }^{20}$ and was measured as maximum metabolic equivalents (METs). Self-reported smoking status was classified as never, ever, or current smoker. Adjustment for length and pattern of follow-up was achieved by including 3 indicator variables (yes/no) in the regression model that represented whether a subject returned each follow-up survey in 1990, 1995, and 1999.

\section{Data Analysis}

Summary isokinetic quadriceps peak torque (QPT) and hamstring peak torque (HPT) values in kilogram-meters were calculated by taking the average of the 6 repetitions ( 3 on right leg; 3 on left leg). As a result of variations in body size, weight, and composition, QPT and HPT values were individualized by dividing each by the subject's body weight (BW) in kilograms (QPT/BW; HPT/BW). Reciprocal muscle group ratios $(\mathrm{H} / \mathrm{Q} \%)$ were calculated using the formula $(\mathrm{HPT} / \mathrm{QPT}) \times 100$. Isotonic $1 \mathrm{RM}$ leg press values were measured in kilograms. QPT/BW, HPT/BW, and leg press values were categorized into gender- and age-specific quartiles: low (bottom 25\%; referent category), moderate (middle 50\%) and high (top 25\%). H/Q\% was classified as low (<55\%), normal (55\% to 65\%; referent category), and high $(>65 \%)$. Gender differences in strength measures and hip or knee osteoarthritis incidence were tested using the Wilcoxon sum rank test and chi-square analyses. Multiple logistic regression was used to calculate sex-specific odds ratios and $95 \%$ confidence intervals. All regression analyses were conducted using SAS software (SAS Institute, Cary, NC) and adjusted for age, body mass index, smoking status, physical activity level, fitness level, and follow-up. The significance level was $P=0.05$ for all analyses.

\section{Results}

Of the 3081 subjects, $7.1 \%$ of men and $7.4 \%$ of women (no significant difference by gender; $P=0.80$ ) reported physician-diagnosed hip or knee osteoarthritis over an average of $14.4 \mathrm{y}$ of follow-up (Table 1). Men with hip or knee osteoarthritis were significantly older, had higher body mass, and were isokinetically weaker than men without osteoarthritis. Men with and without osteoarthritis had similar fitness levels and isotonic leg press strength. Women with osteoarthritis were significantly older, and had greater body mass, less isokinetic quadriceps strength and higher $\mathrm{H} / \mathrm{Q} \%$ than women without osteoarthritis. Women with and without osteoarthritis did not differ in fitness, hamstring strength (HPT:BW), or leg press measures. A significantly lower percentage of men with osteoarthritis were sedentary $(11 \%$ versus $18 \%$ ); the percentage of sedentary women with and without osteoarthritis was not significantly different.

Women with moderate and high quadriceps strength have a significantly reduced risk (55\% to 64\%) of hip or knee osteoarthritis (Table 2). A similar trend $(\sim 25 \%$ reduced risk) is noted among men but was not significant. Hamstring strength was not related to the development of hip or knee osteoarthritis among 
Table 1 Descriptive Characteristics of Study Subjects at Baseline by Gender and Osteoarthritis Status ${ }^{\text {a }}$

\begin{tabular}{|c|c|c|c|c|}
\hline \multirow{3}{*}{$\begin{array}{l}\text { Variable } \\
\text { Age (y) }\end{array}$} & \multicolumn{2}{|c|}{$\begin{array}{c}\text { Men } \\
(n=2422)\end{array}$} & \multicolumn{2}{|c|}{$\begin{array}{l}\text { Women } \\
(n=659)\end{array}$} \\
\hline & $\begin{array}{c}\text { OA } \\
(n=173)\end{array}$ & $\begin{array}{c}\text { No OA } \\
(n=2249)\end{array}$ & $\begin{array}{c}\text { OA } \\
(n=49)\end{array}$ & $\begin{array}{c}\text { No OA } \\
(n=610)\end{array}$ \\
\hline & $49.6 \quad(8.9)^{b}$ & $43.8 \quad(9.6)$ & $49.8 \quad(9.6)^{b}$ & $44.3 \quad(9.8)$ \\
\hline Body mass index & $25.5(3.9)^{b}$ & $24.9 \quad(3.4)$ & $26.0 \quad(4.6)^{\mathrm{b}}$ & $24.8 \quad(3.3)$ \\
\hline Fitness level (METs) & $11.3 \quad(2.5)$ & $11.6 \quad(2.5)$ & $9.2 \quad(1.5)$ & $9.3 \quad(1.8)$ \\
\hline QPT:BW $(\mathrm{kg} / \mathrm{m})$ & $19.5(4.2)^{\mathrm{b}}$ & $21.0 \quad(4.8)$ & $19.1 \quad(5.9)^{\mathrm{b}}$ & $21.0 \quad(4.9)$ \\
\hline HPT:BW (kg/m) & $12.6(2.9)^{b}$ & $13.2 \quad(3.3)$ & $12.6 \quad(3.3)$ & $13.3 \quad(3.5)$ \\
\hline $\mathrm{H} / \mathrm{Q} \%$ & $65.5(12.5)^{b}$ & $64.0(12.6)$ & $68.7(14.6)^{\mathrm{b}}$ & $63.9(12.3)$ \\
\hline Leg press (kg) & $121.7(36.5)$ & $125.0(31.9)$ & $127.9(41.4)$ & 123.0 (31.9) \\
\hline Sedentary $(\%)$ & $11.0^{\mathrm{b}}$ & 18.1 & 16.3 & 13.9 \\
\hline
\end{tabular}

${ }^{a}$ Values represent mean and standard deviation. ${ }^{\mathrm{b}}$ Significant difference $\mathrm{OA}$ versus no $\mathrm{OA}$ at $P \leq 0.05$. OA, self-reported, physician-diagnosed hip or knee osteoarthritis; MET, metabolic equivalent; QPT, average quadriceps peak torque $(\mathrm{kg} / \mathrm{m}) /$ body weight $(\mathrm{kg})$; HPT, average hamstring peak torque $(\mathrm{kg} / \mathrm{m}) /$ body weight $(\mathrm{kg}) ; \mathrm{H} / \mathrm{Q} \%$, hamstring peak torque/quadriceps peak torque $\times 100$.

Table 2 Gender-Specific Association Between Isokinetic Strength Measures and Incident Self-Reported, Physician-Diagnosed Hip or Knee Osteoarthritis

\begin{tabular}{|c|c|c|c|c|}
\hline \multirow{2}{*}{$\begin{array}{l}\text { Strength } \\
\text { measure }\end{array}$} & \multicolumn{2}{|c|}{$\begin{array}{c}\text { Men } \\
(n=2422)\end{array}$} & \multicolumn{2}{|c|}{$\begin{array}{l}\text { Women } \\
(n=659)\end{array}$} \\
\hline & OR & $95 \% \mathrm{CI}$ & OR & $95 \% \mathrm{CI}$ \\
\hline \multicolumn{5}{|l|}{ QPT/BW } \\
\hline Low & 1.00 & Referent & 1.00 & Referent \\
\hline Moderate & 0.74 & $0.52,1.06$ & 0.36 & $0.15,0.90$ \\
\hline High & 0.78 & $0.50,1.23$ & 0.45 & $0.21,0.96$ \\
\hline \multicolumn{5}{|l|}{ HPT/BW } \\
\hline Low & 1.00 & Referent & 1.00 & Referent \\
\hline Moderate & 1.23 & $0.86,1.77$ & 2.65 & $0.96,7.33$ \\
\hline High & 1.00 & $0.62,1.60$ & 1.51 & $0.53,4.28$ \\
\hline \multicolumn{5}{|l|}{$\mathrm{H} / \mathrm{Q} \%$} \\
\hline Low & 1.00 & Referent & 1.00 & Referent \\
\hline Normal & 0.96 & $0.61,1.50$ & 0.81 & $0.33,1.97$ \\
\hline High & 1.50 & $1.03,2.18$ & 1.83 & $0.90,3.71$ \\
\hline
\end{tabular}

OR, odds ratio; CI, confidence interval; QPT, average quadriceps peak torque $(\mathrm{kg} / \mathrm{m}) / \mathrm{body}$ weight $(\mathrm{kg})$; HPT, average hamstring peak torque $(\mathrm{kg} / \mathrm{m}) /$ body weight $(\mathrm{kg}) ; \mathrm{H} / \mathrm{Q} \%$, hamstring peak torque/quadriceps peak torque $\times 100$.

Note. Odds ratios are adjusted for age, body mass index, smoking status, physical activity level, fitness, and length of follow-up. 
Table 3 Gender-Specific Association Between Isotonic One Repetition Maximum Leg Press and Incident Self-Reported, Physician-Diagnosed Hip or Knee Osteoarthritis

\begin{tabular}{|c|c|c|c|c|}
\hline \multirow{2}{*}{$\begin{array}{l}\text { Strength } \\
\text { measure }\end{array}$} & \multicolumn{2}{|c|}{$\begin{array}{c}\text { Men } \\
(n=2422)\end{array}$} & \multicolumn{2}{|c|}{$\begin{array}{l}\text { Women } \\
(n=659)\end{array}$} \\
\hline & OR & $95 \% \mathrm{CI}$ & OR & $95 \% \mathrm{CI}$ \\
\hline \multicolumn{5}{|l|}{ QPT/BW } \\
\hline \multicolumn{5}{|l|}{ Leg press } \\
\hline Low & 1.00 & Referent & 1.00 & Referent \\
\hline Moderate & 0.67 & $0.44,0.99$ & 0.12 & $0.01,1.21$ \\
\hline High & 0.86 & $0.54,1.35$ & 0.28 & $0.08,0.95$ \\
\hline
\end{tabular}

OR, odds ratio; $\mathrm{CI}$, confidence interval

Note. Odds ratios are adjusted for age, body mass index, smoking status, physical activity level, fitness, and length of follow-up.

men or women. A high $\mathrm{H} / \mathrm{Q} \%$ was associated with a $50 \%$ increased risk of osteoarthritis among men, however, there was no significant association between $\mathrm{H} / \mathrm{Q} \%$ and osteoarthritis among women. Moderate global lower extremity strength as measured by a 1RM leg press test was associated with $33 \%$ decreased risk of osteoarthritis among men while women classified as high strength had a $72 \%$ decreased risk (Table 3).

\section{Discussion}

The results of this study are in accord with those of Slemenda et al. ${ }^{17}$ and suggest that quadriceps weakness is an independent risk factor for lower extremity osteoarthritis. This seems to be particularly true among women and not necessarily among men. Identifying modifiable risk factors for lower extremity osteoarthritis is an important goal and has primary prevention implications. Identifying persons with low quadriceps strength at a young or middle age who can be targeted for resistance training programs might potentially reduce their risk of developing lower extremity osteoarthritis and resulting disability.

The actual pathological mechanisms responsible for how quadriceps weakness contributes to the development of lower extremity osteoarthritis are still unclear but are likely multidimensional. It is possible that pain-related disuse atrophy could cause the joint surfaces to be compressed with weight-bearing activity leading to cartilage damage. Slemenda et al. ${ }^{17}$ argues that if this were true, disuse atrophy and muscle weakness would be evident in the entire thigh musculature, which was not evident in either their or our study. Recent information suggests that neuromuscular inhibition of the quadriceps and proprioceptive deficits could be implicated in lower extremity osteoarthritis..$^{3-4,21-22}$ Skeletal muscle plays an integral role in functional joint stability, shock absorbtion, proprioception, and functional movement. Muscle 
dysfunction can result in excessive forces transmitted to the load-bearing joint surfaces, increased joint laxity during dynamic movement, and injury caused by improper neurological input and response. All these have been associated with microtrauma to articular cartilage and subchondral bone and the initiation of the osteoarthritis disease process. ${ }^{23}$ Lastly, joint injury is a known risk factor for lower extremity osteoarthritis ${ }^{24}$ and muscular strength might protect against certain types of joint injuries.

Recent advances in our understanding of gender-related injury patterns could also provide insight into why this relationship between quadriceps weakness and osteoarthritis might only be found among women. ${ }^{25-28}$ Females have significantly lower quadriceps and hamstring strength than males, even when strength is normalized to weight. Gender differences in lower extremity kinematics and kinetics, muscle stiffness, postural alignment, and muscle recruitment patterns have also been documented. Muscle strength could provide protection to the joint during dynamic movement by dispersing excess forces away from the joint surfaces. Muscle strength might also influence joint kinematics and kinetics and postural alignment by improving the efficiency of dynamic joint motion control, ultimately influencing the amount of load and deformation transmitted to the soft tissues and cartilage of the joints. Excessive mechanical stress applied to hyaline cartilage, acutely or accumulated over many years, is one of the mechanisms thought to contribute to cartilage degeneration and subsequent osteoarthritis.

The results of this study are subject to several limitations. First, the population in this study is highly educated, mostly male, and predominantly Caucasian. We did not have information on occupation type but suspect that because of the high education level of this cohort most are employed in white-collar-type jobs, making it unlikely that that occupation would be a confounder. The results of this study might not be generalizable to the overall population, however. Second, some subjects who self-report physician-diagnosed hip or knee osteoarthritis might not have the disease. As a result of the prospective study design, subjects' exposure status (quadriceps strength) was measured years before their outcome (osteoarthritis) status was ascertained. Therefore, it is unlikely that subjects were differentially misclassified regarding their osteoarthritis. Subsequently, our risk estimates would be conservative and biased towards the null value. As a result of the high education level in this population, other self-reported disease outcomes (hypertension: $98 \%$ sensitivity, $99 \%$ specificity) have been reliably reported in this cohort ${ }^{29}$ and a small validity study of self-reported OA showed moderate agreement with medical record documentation. Over $89 \%$ of a random selection of 36 participants self-reporting OA had physician-documented OA. ${ }^{19}$ Lastly, as a result of the survey question format, we were unable to separate hip and knee osteoarthritis. Although the quadricep muscles cross both joints, we are unable to determine whether weakness in this muscle group has the same effects at the hip and knee.

This study has multiple strengths that specifically address limitations in prior studies. First, this is only the second longitudinal study investigating quadriceps weakness as an independent risk factor for lower extremity osteoarthritis and our study had considerably more subjects (3081 versus 342 ) who were followed for an average of $14 \mathrm{y}$ (versus $31.3 \mathrm{mo}$ ) than the previous study. ${ }^{17}$ Second, our subjects were not exclusively elderly at baseline and therefore it is unlikely that our subjects 
had significant age-related muscle changes (i.e. sarcopenia) at baseline. Third, we had injury history information from surveys at 2 different timepoints. This information was used to exclude those with any prior lower extremity injury, which could influence their quadriceps strength as well as their risk for osteoarthritis. Fourth, both physical activity and cardiorespiratory fitness effect muscular strength and the study was specifically designed to investigate the effects of physical activity and fitness on health outcomes. Therefore, the measures of physical activity and cardiorespiratory fitness used in our study are quantitative and more precise. Slemenda et al. ${ }^{17}$ did not account for fitness level and used a qualitative measure of physical activity in which subjects graded their activity level compared to that of their peers.

Because Slemenda et al. ${ }^{17}$ did not report risk estimates in their study, our results are not directly comparable. The evidence from both studies, however, suggests that quadriceps weakness could precede the development of osteoarthritis and is not just a clinical feature of the disease. Many risk factors for lower extremity osteoarthritis such as age, gender, genetic profile, etc. are nonmodifiable. In contrast, muscle strength can be improved through resistance training and these changes are possible at any age ${ }^{30}$ The efficacy of resistance training has been clearly established for persons with existing mild to moderate knee osteoarthritis resulting in decreased pain, improved function, and a reduced risk of disability. ${ }^{12-15}$ Resistance training has also been shown to be beneficial for many other segments of the population and is associated with multiple health benefits such as osteoporosis prevention, functional independence, improved glycemic control among people with diabetes, and lower mortality rates. ${ }^{31-33}$ In fact, resistance training has been recognized as such an important aspect of physical fitness, federal and private health agencies recommend all US adults engage in resistance training exercises at least twice per week ${ }^{34-35}$ Resistance training could be an effective and low cost primary prevention intervention for osteoarthritis by addressing both idiopathic primary quadriceps weakness and joint injury prevention. In addition, engaging in resistance training exercise might encourage individuals to engage in other types of physical activity which might reduce the risk of obesity, another modifiable risk factor for lower extremity osteoarthritis.

\section{Acknowledgments}

This study was supported by National Institutes of Health grant NIA AG06945.

\section{References}

1. Fisher N, Pendergast D. Reduced muscle function in patients with osteoarthritis. Scand J Rehabil Med. 1997;29:213-221.

2. Hassan B, Mockett S, Doherty M. Static postural sway, proprioception, and maximal voluntary quadriceps contraction in patients with knee osteoarthritis and normal control subjects. Ann Rheum Dis. 2001;60:612-618.

3. Hurley M. Quadriceps weakness in osteoarthritis. Cur Op Rheumatol. 1998;10: 246-250.

4. Hurley M. The role of muscle weakness in the pathogenesis of osteoarthritis. Rheum Dis Clin North Am. 1999;25:283-298. 
5. Steultjens M, Dekker J, van Baar M, Oostendrop R, Bijlsma J. Muscle strength, pain and disability in patients with osteoarthritis. Clin Rehab. 2001;15:331-341.

6. Arokoski MH, Arokoski JPA, Haara M, Kankaanpaa M, Vesterninen M, Niemitukia LH, Helminen HJ. Hip muscle strength and muscle cross sectional area in men with and without hip osteoarthritis. J Rheumatol. 2002;29(10), 2187-2195.

7. Messier S, Glasser J, Ettinger W, Craven T, Miller M. Declines in strength and balance in older adults with chronic knee pain: a 30-month longitudinal, observational study. Arthritis Rheum. 2002;47:141-148.

8. O'Reilly S, Jones A, Muir K, Doherty M. Quadriceps weakness in knee osteoarthritis: the effect on pain and disability. Ann Rheum Dis. 1998;57:588-594.

9. Pendelton A, Arden N, Dougados M, Doherty M, Bannwarth B, Bijlsma J, et al. EULAR recommendations for the management of knee osteoarthritis: report of a task force of the Standing Committee for International Clinical Studies Including Therapeutic Trials (ESCISIT). Ann Rheum Dis. 2000;59:936-944.

10. Philadelphia Panel. Philadelphia Panel evidence-based clinical practice guidelines on selected rehabilitation interventions for knee pain. Phys Ther. 2001;81:1675-1700.

11. van Baar M, Assendelft W, Dekker J, Oostendrop R, Bijlsma J. Effectiveness of exercise therapy in patients with osteoarthritis of the hip or knee: a systematic review of randomized clinical trials. Arthritis Rheum. 1999;42:1361-1369.

12. Baker K, Nelson M, Felson D, Layne J, Sarno R, Roubenoff R. The efficacy of home based progressive strength training in older adults with knee osteoarthritis: a randomized controlled trial. J Rheumatol. 2001;28:1655-1665.

13. Ettinger, Burns R, Messier S, Applegate W, Rejeski W, Morgan T, et al. A randomized trial comparing aerobic exercise and resistance exercise with a health education program in older adults with knee osteoarthritis. JAMA. 1997;277:25-31.

14. Maurer B, Stern A, Kinossian B, Cook K, Schumacher H. Osteoarthritis of the knee: isokinetic quadriceps exercise versus an educational intervention. Arch Phys Med Rehab. 1999;80:1293-1299.

15. Schilke J, Johnson G, Housh T, O'Dell J. Effects of muscle strength training on the functional status of patients with osteoarthritis of the knee joint. Nurs Res. 1996;45: 68-72.

16. Slemenda C, Brandt K, Heilman D, Mazzuca S, Braunstein E, et al. Quadriceps weakness and osteoarthritis of the knee. Ann Intern Med. 1997;127:97-104.

17. Slemenda C, Heilman D, Brandt K, Katz B, Mazzuca S, Braunstein E, et al. Reduced quadriceps strength relative to body weight: a risk factor for knee osteoarthritis in women? Arthritis Rheum. 1998;41:1951-1959.

18. Evans W, Campbell W. Sarcopenia and age-related changes in body composition and functional capacity. J Nutr. 1993;123:465-468.

19. Cheng Y, Macera C, Davis D, Ainsworth B, Troped P, Blair S. Physical activity and self-reported, physician diagnosed osteoarthritis: is physical activity a risk factor? J Clin Epidemiol. 2000;53:315-322.

20. Pollock M, Bohannon R, Cooper K, et al. A comparative analysis of four protocols for maximal treadmill stress testing. Am Heart J. 1976;92:39-46.

21. Sharma L. Proprioceptive impairment in knee osteoarthritis. Rheum Dis Clin North Am. 1999;25:299-314.

22. Suter E, Herzog W. Does muscle inhibition after knee injury increase the risk of osteoarthritis? Exerc Sport Sci Rev. 2000;28:15-18. 
23. Whitting W, Zernicke R. Tissue biomechanics and adaptation. In: Biomechanics of Musculoskeletal Injury. Champaign, IL: Human Kinetics, 1998:87-112.

24. Felson D, Lawrence R, Dieppe P, Hirsch R, Helmick C, Jordan J, et al. Osteoarthritis: new insights. Part I: The disease and its risk factors. Ann Intern Med. 2000;133:635646.

25. Griffin LY, Agel J, Alhohm MJ, Arendt EA, Dick RW, Garrett WE, et al. Noncontact anterior cruciate ligament injuries: risk factors and prevention strategies. $J$ Am Acad Orthop Surg. 2000;8(3):141-150.

26. Henry JC, Kaeding C. Neuromuscular differences between male and female athletes. Curr Womens Health Rep. 2001;1(3):241-244.

27. Huston LJ, Greenfield ML, Wojtys EM. Anterior cruciate ligament injuries in the female athlete. Potential risk factors. Clin Orthop. 2000;372:50-63.

28. Lephart SM, Abt JP, Ferris CM. Neuromuscular contributions to anterior cruciate ligament injuries in females. Curr Opin Rheumatol. 2000;14(2):168-173.

29. Blair SN, Goodyear N, Gibbons L, Cooper K. Physical fitness and incidence of hypertension in healthy normotensive men and women. JAMA. 1984;252(4):487-490.

30. Roth S, Ferrell R, Hurley B. Strength training for the prevention and treatment of sarcopenia. J Nutri Health Aging. 2000;4:143-155.

31. Kell R, Bell G, Quinney A. Musculoskeletal fitness, health outcomes and quality of life. Sport Med. 2001;31:863-873.

32. Winett R, Carpinelli R. Potential health-related benefits of resistance training. Prev Med. 2001;33:503-513.

33. FitzGerald SJ, Barlow CE, Kampert JB, Morrow JR, Jackson AW, Blair SN. Muscular fitness and all-cause mortality: prospective observations. J Phys Activity Health. 2004;1:7-18.

34. American College of Sports Medicine. The American College of Sports Medicine Position Stand: the recommended quantity and quality of exercise for developing and maintaining cardiorespiratory and muscular fitness, and flexibility in healthy adults. Med Sci Sport Exerc. 1998;30:975-991.

35. Department of Health and Human Services. Healthy People 2010: Objectives for Improving Health. Washington, DC: U.S. Government Printing Office, 2000; pp.22.1322.17 . 